\title{
Findings from high resolution computed tomography of the lung and pleura of symptom free workers exposed to amosite who had normal chest radiographs and pulmonary function tests
}

\author{
Silvano Neri, Alessandro Antonelli, Fabio Falaschi, Piero Boraschi, Lidio Baschieri
}

\begin{abstract}
The lungs of 50 symptom free workers exposed to amosite and with normal pulmonary function tests were examined by high resolution computed tomography (HRCT). Twenty five had normal standard chest radiographs whereas the other 25 had radiographs interpreted as near normal (International Labour Office profusion score $<0 / 1$ or suspected pleural plaques). In 13 of the workers the results of HRCT were negative; in 22 pleural plaques were found, in five there was only parenchymal involvement, and 10 had both pleural and parenchymal changes. The mean duration of exposure to amosite was significantly longer for the subjects with parenchymal signs than for those with normal parenchyma and for the workers with pleural plaques than for those with normal pleura and lung parenchyma. The prevalence of identified pleural and parenchymal abnormalities in the 50 workers was also significantly higher than in a reference group without exposure to asbestos. It is concluded that HRCT may detect initial lung and pleural involvement in symptom free workers exposed to amosite and the mean duration of exposure is longer for subjects with parenchymal or pleural involvement.
\end{abstract}

(Occup Environ Med 1994;51:239-243)

Pulmonary diseases related to asbestos are important medical concerns and legal issues. In the absence of histological examination the clinical diagnosis of asbestosis in workers exposed to asbestos is currently based on the following criteria ${ }^{1}$ : (1) chest radiographic evidence of small irregular opacities with a profusion of at least $1 / 1$ according to the International Labour Office classification ${ }^{2}$; (2) a restrictive pattern of lung impairment; (3) a reduction of $\mathrm{CO}$ diffusing capacity; (4) the presence of bilateral late or paninspiratory crackles on auscultation at the posterior lung bases not cleared by cough. The findings from chest radiography are thought to be the most important and the presence of pleural abnormalities could help to indicate that a patient with pulmonary fibrosis has asbestosis. It is well known that chest radiographs may underestimate the presence of both interstitial lung involvement and pleural plaques. In fact pathological changes of asbestosis $^{3-5}$ have been found in the absence of radiographically evident fibrosis or abnormalities of pulmonary function. ${ }^{6}$ The issue of the earliest lesions of asbestosis and their significance remains under debate. ${ }^{78}$ High resolution computed tomography (HRCT) has now provided a sensitive tool to show initial lung parenchymal fibrosis and pleural asbestos related lesions in symptomatic workers exposed to asbestos, ${ }^{9}$ even when standard chest radiographs are negative. The specificity of the HRCT findings in asbestosis is, however, still a matter of controversy.

The aim of our perspective study was to investigate the possibility of detecting early asbestos related lung and pleural lesions within a cohort of symptom free workers exposed to amosite who had negative chest radiographs, and $\mathrm{CO}$ diffusing capacity and pulmonary function tests in the normal range. Furthermore we evaluated the relation between the duration of exposure to amosite and the prevalence of abnormalities detected by HRCT in the study group and the specificity of HRCT findings, by comparison of the exposed group with a control group composed of patients without any known professional exposure to asbestos.

\section{Methods}

All the subjects of the study group worked in a factory producing naval furniture where one case of mesothelioma ${ }^{10}$ had been previously found by the sentinel health event method. In this factory a material containing about $40 \%$ amosite was used, from 1958 to 1980 . This was especially in the carpentry department where panels were built consisting of asbestos insulation between two external layers of wood. The study group comprised 127 workers whose chest radiographs, performed according to ILO criteria, were previously judged as negative by outside readers. Three radiologists experienced in the reading of radiographs according to the ILO classification have re-examined the 127 standard chest radiographs.

Among the 127 workers we selected the subjects who fitted the following criteria: (1) documented occupational exposure to asbestos; (2) absence of any clinical symptomatology 
Table Results of pulmonary function tests (\% of predicted values) in workers with negative or positive chest radiograph

\begin{tabular}{|c|c|c|c|c|c|}
\hline & $T L C$ & $V C$ & $F E V_{1}$ & $F E V_{1} / F V C \%$ & $T L C O s b$ \\
\hline $\begin{array}{l}\text { Negative } \\
\text { Positive }\end{array}$ & $\begin{array}{r}98 \cdot 3(9 \cdot 2) \\
101 \cdot 6(7 \cdot 1)\end{array}$ & $\begin{array}{l}96.5(5.2) \\
98.4(8.6)\end{array}$ & $\begin{array}{l}94 \cdot 7(7 \cdot 9) \\
92 \cdot 8(8 \cdot 3)\end{array}$ & $\begin{array}{l}98 \cdot 6(6 \cdot 3) \\
96 \cdot 2(4 \cdot 7)\end{array}$ & $\begin{array}{l}118.4(11.3) \\
123.2(21.2)\end{array}$ \\
\hline
\end{tabular}

Results are means (SD); TLC = total lung capacity; VC = vital capacity; FEV $_{1}=$ forced expiratory volume in one second; FVC $=$ forced vital capacity; TLCO $=$ carbon monoxide transfer factor.

suggestive of asbestosis or of any other lung disease; (3) normal pulmonary function tests (TLC, VC, FVC, $\mathrm{FEV}_{1}, \mathrm{FEV}_{1} / \mathrm{FVC}$ ), and TLCo (single breath) (see table) not more than four weeks before HRCT; pulmonary function tests were performed by HP 47120 A equipment that meets American Thoracic Society recommendations ${ }^{11}$ and reference values were obtained from previous Italian studies $^{12}{ }^{13}$; (4) workers' consent to perform the HRCT.

Among this group we selected 50 workers: 25 who had a plain chest radiograph confirmed negative, both for pleura and pulmonary parenchyma, by our three independent radiologists and 25 with radiographs for which an ILO profusion score of $0 / 1$ or pleural plaques were suspected by two or three of our readers and who were considered "positive". The mean age of the 50 subjects (49 men) was $45 \cdot 7$ (SD 6.9) (range: $31-61$ ) years.

Occupational history was obtained by direct interview and by the evaluation of every document available from the factory medical service before HRCT examinations. Exposure is expressed in cumulative years.

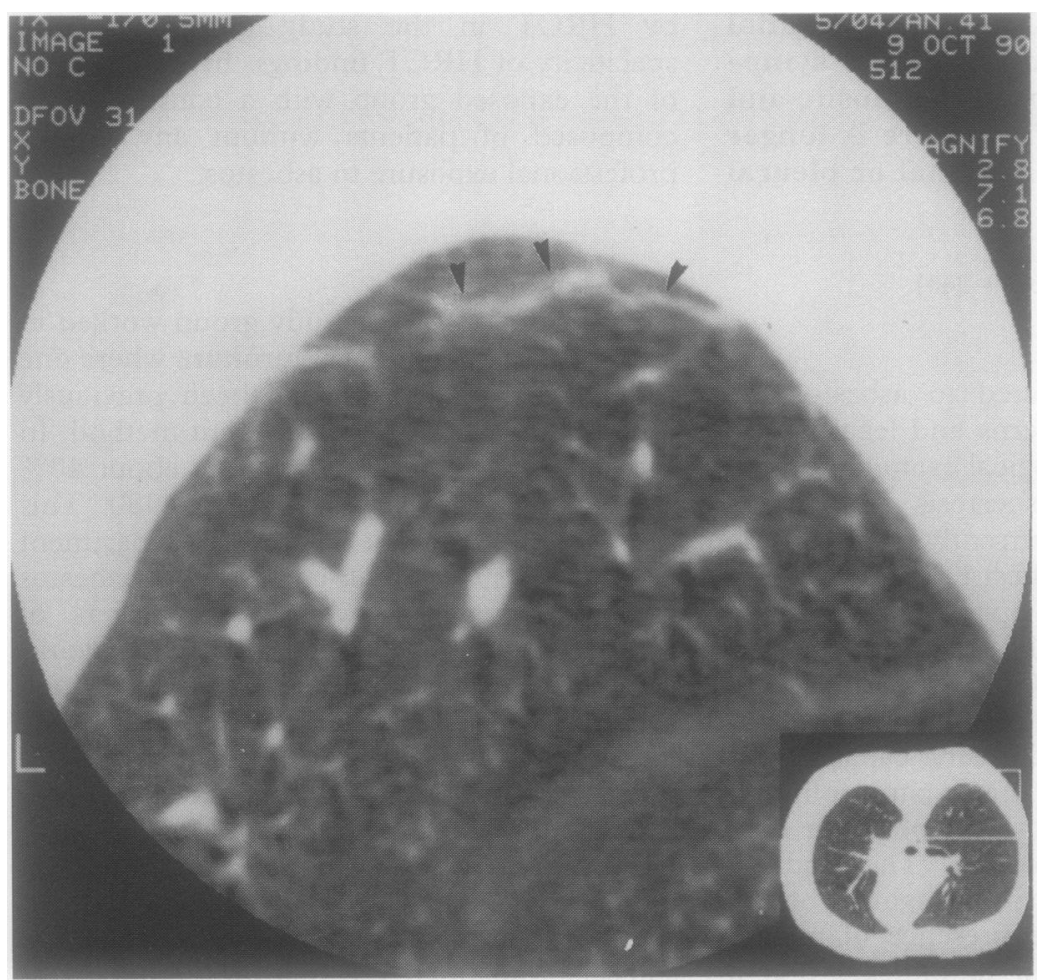

Figure 1 Prone HRCT scan showing a subpleural curvilinear shadow parallel to inner chest wall (see arrows).
Individual health histories were also investigated: none of the workers had a history of pleurisy, empyema, haemothorax, tuberculosis treated with pneumothorax, or other pulmonary diseases.

The interval between HRCT and standard chest radiograph varied from 0.2 to 19 months (mean interval 4.4 (3.8) months). The HRCT study was performed on a GE-9800 scanner. All patients were examined at suspended maximum inspiration, both in the supine position and prone when posteroinferior portions of the lungs showed obscuration dependent on gravity attenuation or any of the HRCT abnormalities associated with asbestosis were identified. HRCT sections $1.5 \mathrm{~mm}$ thick were made at $20 \mathrm{~mm}$ intervals from the aortic arch to the diaphragm. To minimise the noise $140 \mathrm{KVp}$ and $170 \mathrm{mAs}$ were used, with 2.5 seconds of acquisition time. The image was finally obtained through a reconstruction with a convolution filter of the bone detail algorythm. FoV was $24 / 30 \mathrm{~cm}$. All images were photographed at the $500 \mathrm{HU}$ window for pleura and mediastin and $1500 \mathrm{HU}$ window for pulmonary parenchyma. HRCT scans were interpreted by a radiologist blinded to individual exposure data and to chest radiographic interpretation. Pleural alterations were quantified applying 1980 ILO criteria to the reading of CT scans. Parenchymal abnormalities were interpreted on the bases of published data ${ }^{14}$ and were considered diagnostic of asbestosis when present in a combination of findings (two or more).

HRCT findings have been related to the individual exposure data and compared with those of a reference group of 20 hospital patients (mean age $47 \cdot 1(13 \cdot 4)$, range $26-67$ years; 19 men) who were given HRCT for suspected localised pulmonary pathology (in most of cases nodules cysts, or embolus) not confirmed by HRCT, without occupational or significant environmental exposure to asbestos.

Smoking histories were not different for the subjects exposed to asbestos with negative or positive chest radiographs and the control group (pack-years respectively $12 \cdot 1(9 \cdot 2)$, $13 \cdot 6(8 \cdot 4)$, and $14 \cdot 8(9 \cdot 7))$.

Statistical analysis was by analysis of variance (ANOVA-Fisher PLSD), independent $t$ test (two tailed), or contingency table analysis, as indicated. The results are expressed as means (SD). A statistically significant level was considered to be present at $\mathrm{p}<0.05$.

The protocol of this study was approved by the University Ethics Committee for Human Studies.

\section{Results}

Among the 50 workers examined by HRCT, 22 showed pleural plaques, five exclusively parenchymal abnormalities, and 10 both pleural and parenchymal abnormalities; in the other 13 workers no pathological finding was shown. 


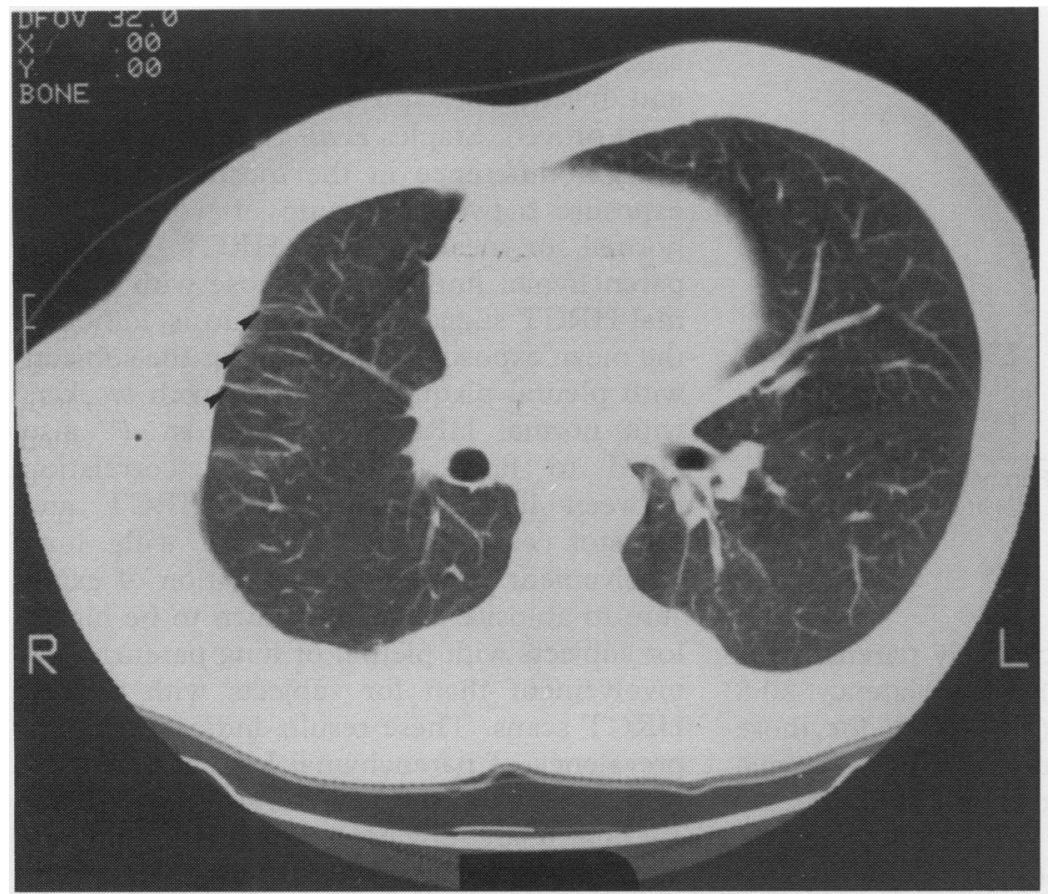

Figure 2 HRCT scan photographed at a lung window, showing thickened interlobular septae in the right lung (see arrows).

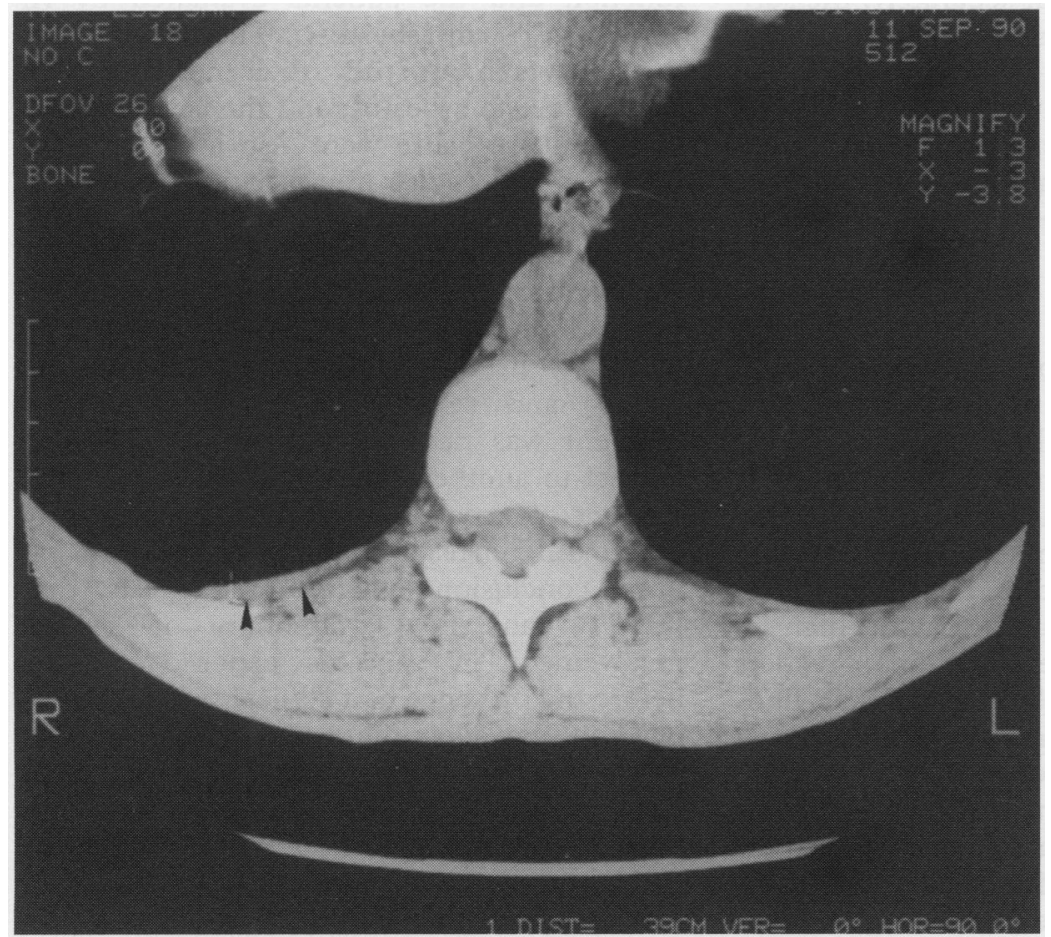

Figure 3 HRCT scan, photographed at a mediastinal window, showing measure of pleural thickening in the costovertebral space (see arrows).

Figure 4 Prevalence (by HRCT) of normal findings, and pleural, parenchymal, and both pleural and parenchymal lesions among workers exposed to amosite and controls.

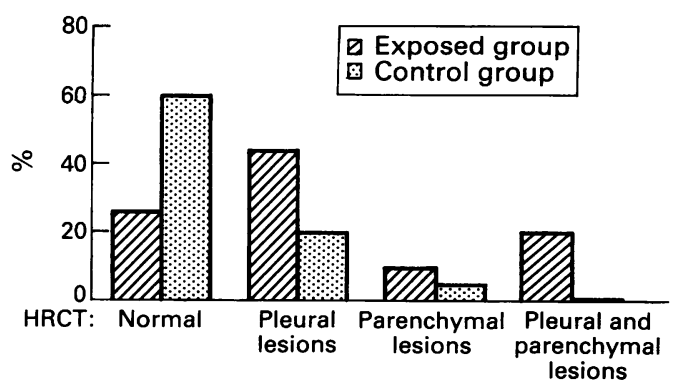

The parenchymal abnormalities were: subpleural curvilinear lines (eight cases; fig 1); thickened interlobular (septal) lines (three cases; fig 2); hazy patches of increased attenuation (six cases); and thickened intralobular (core) lines (three cases). In four workers two or more of the described signs were found. No case of honeycombing was reported.

Thirty two workers had pleural plaques (fig 3) (23 of them bilateral). Only one worker showed diffuse pleural thickening (the same subject also had interstitial abnormalities). No one had benign exudative pleurisy. In nine subjects, plaques were calcified. Plaques located in the tendinous part of the diaphragm were present in 21 cases (one of these had exclusively diaphragmatic plaques).

Among the 25 subjects whose standard radiographs were confirmed "negative" by our radiologists, HRCT showed 11 cases of pleural plaques, two cases with both pleural and parenchymal involvement, and two cases with only a subpleural curvilinear shadow, which is not specific for asbestosis as an isolated finding. Among the workers for whom pleural plaques were suggested at standard chest radiographic reading (18 cases), HRCT confirmed these in 16 cases, in five of whom HRCT also showed parenchymal involvement; in two cases pleural plaques were not confirmed and no pathological finding emerged. HRCT confirmed parenchymal involvement for five of the six subjects whose radiographs showed parenchymal opacities with a profusion $0 / 1$ and in two of these cases not shown as having pleural plaques; in one case no pathological finding was shown and in one case a standard radiograph and HRCT showed both pleural and parenchymal involvement.

Sensitivity, specificity, accuracy, and positive and negative predictive values of standard chest radiographs were calculated with respect to HRCT (artificially assuming it as gold standard) and resulted in, respectively, for pleural and parenchymal findings: sensitivity $89 \%$ and $40 \%$; specificity $53 \%$ and $97 \%$; accuracy $66 \%$ and $80 \%$; negative predictive value $52 \%$ and $79 \%$; and positive predictive value $89 \%$ and $86 \%$.

In the control group (20 subjects) HRCT identified five cases with pleural plaques (only in one case bilaterally; all these were $1 \mathrm{~A}$ on the ILO scale); parenchymal bands were found in one subject. Lung and pleural lesions detected by HRCT in the control group were significantly less than those found among the group exposed to amosite (contingency table analysis: $p<0.001$; fig 4 ).

The mean duration of exposure of the whole group of workers exposed to amosite was $6 \cdot 4(5 \cdot 5)$ (range: $0 \cdot 2-21$ ) years and the mean latency since the time of first exposure to the examination was $20.7(5 \cdot 0)$ years. In the different subgroups the mean duration of exposure (fig 5) was $2.3(3.0)$ years for subjects with normal HRCT (group 1); mean latency $18.3(5 \cdot 2)$ years; $7 \cdot 0(5 \cdot 5)$ years for the workers with pleural alterations (group 2); mean latency $21 \cdot 1(4 \cdot 7)$ years; $5 \cdot 2(3 \cdot 0)$ 
Figure 5 Mean (bar $=$ SD) duration of exposure for the subgroups of workers (defined on the bases of HRCT results).

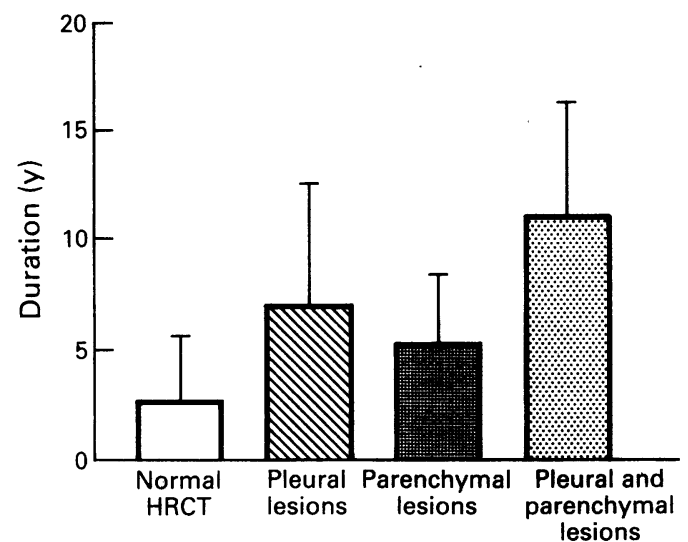

years for those with exclusively parenchymal abnormalities (group 3); mean latency 20.8 $(6.1)$ years; and $11.0(5 \cdot 2)$ years for those who presented both pleural and parenchymal involvement (group 4); mean latency 23.0 $(4 \cdot 0)$ years.

The duration of exposure to amosite for the workers of group 4 and group 2 was significantly longer than for the workers of group 1 (ANOVA Fisher PLSD: $p<0.005$ and $\mathrm{p}<0.05$, respectively). Also, considering parenchymal involvement separately, the subjects with HRCT findings suggestive of pulmonary fibrosis (group $3+$ group 4) had been exposed to amosite for a longer time than workers with normal parenchyma (group $1+$ group 2): 9.1 years $v 5.3$ years $(p<$ 0.05 ; $t$ independent test).

\section{Discussion}

HRCT of the lung is probably the most sensitive current radiological means to detect early parenchymal and pleural asbestos related diseases, and has proved to be more sensitive than chest radiographs ${ }^{15}$ or conventional CT. ${ }^{16}$ Our data confirm that HRCT is very sensitive and suggest that the accuracy of standard chest radiographs is not high in symptom free workers even if expert readers are involved. The specificity of HRCT findings seems to be confirmed by the high prevalence of detected lesions among workers exposed to asbestos ${ }^{9}$ and by the proof that HRCT findings correlate well with pathological findings, ${ }^{17}$ even if it remains a controversial issue due to the limited number of correlative pathological studies. Our data from the symptom free amosite exposed workers in comparison with the controls suggest a probable relation between HRCT findings and exposure to asbestos. Pleural and parenchymal findings among controls may be due to causes other than exposure to asbestos or a possible occupational exposure to asbestos not known from individual health histories. The presence of low levels of asbestos fibres in the general environment also seems to be related to development of parenchymal or pleural abnormalities. Whereas exposure-response relations for the major asbestos related diseases have been considered demonstrable, ${ }^{18}$ the correlation between the early lung lesions related to asbestos as detected by HRCT and the levels and duration of exposure has actually not yet been proved. Staples $e t a l^{9}$ did not find a significant difference in the mean duration of exposure between a group of workers with normal or near normal HRCT for lung parenchymal findings and those with abnormal HRCT suggestive of asbestosis, although the mean exposure was higher for the subjects with pleural plaques compared with workers with normal HRCT. Al Jarad et $a l^{19}$ also failed to find any significant correlation between lung findings from HRCT and asbestos exposure in patients with lung involvement. In our study duration of exposure to amosite has been shown to be higher for subjects with pleural or lung parenchymal involvement than for subjects with normal HRCT scans. These results indicate that the prevalence of parenchymal lesions shown by HRCT and the severity of lung involvement are dose related, by contrast with the previous findings. There are various explanations for the discrepancy. Our subjects were clinically symptom free whereas many of the subjects evaluated by Staples et $a l^{9}$ presented with dyspnoea or abnormalities of pulmonary function and the patients evaluated by $\mathrm{Al}$ Jarad et $a l^{19}$ had asbestosis. The mean age of the subjects in our study, their mean duration of exposure to asbestos, and the latency times since first exposure were also lower than in the previous studies; these data may explain why in our cohort only four cases of radiologically confirmed asbestosis were present. Furthermore our sample of workers had been exposed to the same type of asbestos fibre (amosite) in the same occupations, so the quality and severity of exposure at any moment was not much different from one subject to another, and the differences in the amount of exposure were almost exclusively due to differences in duration of exposure.

In conclusion our study suggests that HRCT can show pulmonary or pleural involvement in asbestos exposed workers before the presence of a clinical symptomatology and may allow the detection of asbestosis in some clinically symptom free workers. In fact in our study HRCT showed a combination of parenchymal abnormalities sufficient to be considered diagnostic for asbestosis in four symptom free subjects with normal pulmonary function tests and diffusing capacity (in a preclinical phase). Furthermore, 11 cases of pleural plaques and two cases with both pleural and parenchymal involvement were found by means of HRCT among the 25 workers with normal chest radiographs. The lung and pleural abnormalities detected by HRCT in our sample of symptom free workers seem to be related to asbestos exposure also in the subjects with negative or equivocal chest radiographs, as in most clinically evident asbestos related lung diseases. ${ }^{20}$ The relevance of isolated slight pleural or parenchymal HRCT findings in the clinically symptom free workers with normal pulmonary function tests is not yet clear and 
there is no evidence that isolated parenchymal or pleural findings may progress and produce clinical disability. We suggest a careful surveillance of these workers, however, and we propose to re-evaluate them after a period long enough to assess the progression of such abnormalities.

1 American Thoracic Society-Medical Section of the American Lung Association. The diagnosis of nonmalignant diseases

2 International Labour Office. Guidlines for the use of $I 10$ international classification of radiographs of pneumoconioses: revised 1980. Geneva; IIO 1980. (Occupational safety and health series No 22.)

3 Rockoff SD, Schwartz A. Roentgenographic underestimation of early asbestosis by International Labor Organization classification. Analysis of data and probabilities. Chest 1988;93:1088-91.

4 Kippen HM, Lilis R, Suzuki Y, Valciukas JA, Selikoff IJ Pulmonary fibrosis in asbestos insulation workers with Pulmonary fibrosis in asbestos insulation workers with lung cancer: a radiological and histopa

5 Rubino GF, Scansetti G, Pira E, et al. Pleural plaques and lung asbestos bodies in the general population: an autoptical and clinical radiological survey. IARC Sci Publ 1980;30:545-51.

6 Bader ME, Mader RA, Tierstein AS, Miller A, Selikoff IJ Pulmonary function and radiographic changes in 598 workers with varying duration of exposure to asbestos. Mt Sinai 7 Med 1970;37:492-500.

7 Churg A. The diagnosis of asbestosis. Hum Pathol 1989; 20:97-9.

Shith is asbestosis? Chest 1990;98:953-4

9 Staples CA, Gamsu G, Ray CS, Webb WR. High resolution computed tomography and lung function in asbestos-exposed workers with normal chest radioasbestos-exposed workers with normal che

10 Neri S, Iaia TE, Roselli MG, Battista G. Gli eventi sentinella in medicina del lavoro: l'esempio dell'USL 1 della Regione Toscana. Epidemiol Prev 1989;39:29-34.

11 American Thoracic Society. Standardization of spirometry: 1987 update. Am Rev Respir Dis 1987;136: $1285-98$.

12 Paoletti P, Pistelli G, Fazzi P, et al. Reference values for vital capacity and flow-volume curves from a general population study. Bullettin Europeen de Physiopatologie Respiratoire 1986;22:451-9.

13 Paoletti $P$, Viegi G, Pistelli G, et al. Reference equations for the single breath diffusing capacity. A cross sectional analysis and effect of body and size. Am Rev Respir Dis analysis and effect

14 Aberle DR, Gamsu G, Ray CS, Feuerstein IM. Asbestos related pleural and parenchymal fibrosis. Detection with high-resolution CT. Radiology 1988;166:729-34.

15 Friedman AC, Fiel SB, Fisher MS, Radecki PD, LevToaff AS, Caroline DF. Asbestos-related pleural disease and asbestosis: a comparison of $\mathrm{CT}$ and chest radiography. American foumal of Roentgenology 1988;150: 269-75.

16 Lynch DA, Gamsu G, Aberle DR. Conventional and high resolution computed tomography in the diagnosis of resolution computed tomography in the diagnosis of

17 Akira M, Yamamoto S, Yokoyama K, et al: Asbestosis: high-resolution CT-pathologic correlation. Radiology 1990;176:389-94

18 Becklake MR. Asbestos-related diseases of the lungs and other organs: their epidemiology and implications fo clinical practise. Am Rev Respir Dis 1976;114:187-227.

19 Al Jarad N, Poulakis N, Pearson MC, Rubens MB, Rudd RM. Assessment of asbestos-induced pleural disease by computed tomography: correlation with chest radiograph and lung function. Respir Med 1991;85:203-8.

20 Becklake MR. Asbestos-related diseases of the lung and pleura: current clinical issues. Am Rev Respir Dis 1982. 126:187-94.

\section{Correspondence and editorials}

Occupational and Environmental Medicine welcomes correspondence relating to any of the material appearing in the journal. Results from preliminary or small scale studies may also be published in the correspondence column if this seems appropriate. Letters should be not more than 500 words in length and contain a minimum of references. Tables and figures should be kept to an absolute minimum. Letters are accepted on the understanding that they may be subject to editorial revision and shortening.

The journal also publishes editorials which are normally specially commissioned. The Editor welcomes suggestions regarding suitable topics; those wishing to submit an editorial, however, should do so only after discussion with the Editor. 